\title{
Partial-Information-Based Synchronization of Complex Networks with Multiple and Event-Triggered Couplings
}

\author{
Chi Huang $\mathbb{D}^{1}{ }^{1}$ Yuning Xiong $\mathbb{D}^{1}$, and Wei Wang $\mathbb{D}^{2}$ \\ ${ }^{1}$ School of Economic Information Engineering, Southwestern University of Finance and Economics, Chengdu 611130, China \\ ${ }^{2}$ College of Data Sciences, Taiyuan University of Technology, Taiyuan 030024, China \\ Correspondence should be addressed to Chi Huang; huangchi@swufe.edu.cn
}

Received 24 December 2020; Revised 25 March 2021; Accepted 7 April 2021; Published 19 April 2021

Academic Editor: Peng Ji

Copyright (c) 2021 Chi Huang et al. This is an open access article distributed under the Creative Commons Attribution License, which permits unrestricted use, distribution, and reproduction in any medium, provided the original work is properly cited.

We study the synchronization of complex networks by using event-sampling information. The nodes of the network are connected with event-triggered communication via multiple couplings. The couplings are split into several channels. Not all the channels are connected. Only a part of the states of each node can be communicated by the channels. An event detector is designed for each channel to independently determine the sampling moments. The couplings of the network are partial and event-triggered. Both features make that less information can be used for synchronization. The pinning controllers are also designed based on the sampled information. By establishing a time-dependent Lyapunov functional and utilizing an efficient event condition, we derive less conservative criteria for the synchronization of complex networks. Finally, the effectiveness of our main results is verified by an illustrative example, and comparisons are also presented to show how much conservatism can be reduced.

\section{Introduction}

During the last decades, the research of complex networks has attracted an increasing amount of attention. Without the central control, the network can perform many collective behaviors just through the local communications among adjacent neighbors. Hence, complex networks have extensive applications in many large-scale systems, such as biology, circuit systems, robotics, and unmanned vehicles. Among all the collective behaviors, synchronization is of great significance due to its wide existence in the real world, for example, fireflies and the school of fish. Recently, the synchronization of complex networks has been intensively studied. Many excellent synchronization results have also been derived in [1-4].

The key to realizing synchronization is the connections among nodes. In earlier research, more of a concern was to investigate the structural topology of complex networks [5-8]. More recently, the focus of the research has shifted to the communication environment [9]. With the rapid development and spread of digital technologies, natural signals are analog and have to be converted into digital format to be used in electronic equipment. It is referred to as the sampling issue. Initially, the time-triggered sampling protocol was widely employed [10]. The main feature of time-triggered communication is that the occurrence of sampling is determined by a predefined time sequence. The time-triggered sampling has its advantage in fault-tolerant systems since the missing data can be detected immediately. However, it is a lack of flexibility and the restrictive design process [11]. All the sampling processes have to be manipulated strictly according to the time sequence. It is hard to dynamically regulate the sampling plan based on the system's real-time performance. Therefore, the time-triggered sampling usually leads to a conservative usage of the communication resource. Another drawback of time-triggered sampling is that all components of a system should be timely synchronous during operation to guarantee the strict timing specification of the system. Thus, time-triggered sampling is hard to be carried out in a large-scale system, especially for complex networks. To overcome the deficiencies of time-triggered sampling, event-triggered sampling has been introduced. It is kind of a real-time scheduling algorithm. An event condition is designed to decide sampling instants. When the 
condition cannot be satisfied, the state of the system needs to be measured. Compared with the time-triggered sampling, the event-triggered counterpart can quickly react to sudden fluctuations [12]. Hence, it is considered a more competent sampling protocol. Due to its virtue, numerous attention has been drawn to this subject, which includes the stabilizing control tasks of single systems $[13,14]$ and the consensus issues of networked systems, such as multiagent systems $[15,16]$, complex networks $[17,18]$, coupled neural networks [19-21], sensor networks [22], and Boolean networks [23-27].

Each connection of complex networks consists of several channels to transmit distinct levels of node's information. In most existing works, all these channels can work properly. It is not realistic for many real systems. In mammalian brain networks, neurons in the same cortical area interact more intensively. However, only a small part of neurons (about $5 \%)$ can receive excitatory synapses to neurons from connected cortical areas $[28,29]$. It indicates that communication among cortical areas counts on the partial couplings which are established by a fraction of neurons. Thus, it is necessary to study the partial couplings of complex networks. As it is known, there are two types of neuronal signals: electrical or chemical signals. Some of the neurons may send electrical signals along with the fundamental chemical signals, while some others may only send one kind of signal. The couplings among complex networks should have multiple layers to communicate different signals. Furthermore, the couplings belonged distinct layers are also partially connected via various parts of a node. Thus, a more realistic communication environment is reflected by the couplings considered in this work, which are multiple partial and obeyed to the event-triggered update.

Despite the theoretical and practical significance, a few results have been proposed for synchronization of complex networks with multiple partial and event-triggered couplings. The difficulties of this problem can be categorized as three aspects. Firstly, the sampling scheme and partial connection lead to less information available for communication. The mechanisms of them are different. The sampling makes node's information available at a sequence of discrete instants. It is a "time-dependent" loss of information. On the other hand, the partial connection means that only a part of the node's information is transmitted, which can be seemed like a "space-dependent" loss. Double reductions make that much less information can be used for synchronization. Secondly, channels of any connection sample information separately, while they need to cooperatively converge. Thus, the event detector of each channel should be designed in a distributed way to realize a global object. Furthermore, the information used for design should also be sampled and partial. Thirdly, the sampled data is in essence a delayed signal [30]. Due to the complicated behaviors of a single node, the conservatism of synchronization criteria is unavoidable. It is necessary yet difficult to reduce the conservatism to make the conditions more applicable.

This work will address the partial-information-based synchronization of complex networks with multiple and event-triggered couplings. The event-triggered sampling is considered for every channel of each connection, in which an event detector is designed based on the sampled and partial information. The event detector individually determines the sampling moment of the corresponding channel. A unified framework will be built to cope with the two kinds of information loss. Channels are rearranged according to their levels of connection. Pinning controllers are also deployed for a small fraction of nodes. The sampled data is used to build the pinning controllers. Since the sampled signal has a time delay, it is hard to eliminate the conservatism of the proposed synchronization criteria. To reduce the conservatism, a delay-dependent Lyapunov functional will be constructed. Finally, a simulative example is proposed to illustrate the efficiency and effectiveness of our results.

Notations. $\mathbb{N}$ is the set of nonnegative integers. $\mathbb{R}^{n}$ and $\mathbb{R}^{n \times n}$ represent, respectively, the $n$ dimensional Euclidean space and the set of $n \times n$ real matrices. $I_{n}, 0$ denote the identity matrix of size $n$ and the zero matrix with proper dimensions, respectively. For real symmetric matrices $X$ and $Y$, the notation $X<Y$ (respectively, $X \leq Y$ ) indicates that the matrix $X-Y$ is negative definite (respectively, seminegative definite). For any two integers $a \leq b$, the notation $[a: b]$ is the set $\{x \in \mathbb{N} \mid a \leq x \leq b\}$. $\|\cdot\|$ denotes the Euclidean norm for a real vector. The superscript " $T$ " represents the transpose of a matrix. $\operatorname{diag}\{\cdots\}$ denotes a diagonal matrix. $\lambda_{\min }(A)$ denotes the smallest eigenvalues of a matrix $A$. Matrices, if not explicitly stated, are assumed to have compatible dimensions. The symmetric term in a matrix is denoted by $*$.

\section{Problem Formulation and Preliminaries}

The dynamics of a node of a complex network can be described as

$$
\dot{x}_{i}(t)=C x_{i}(t)+\mathrm{BF}\left(x_{i}(t)\right)+\alpha w_{i}(t)+u_{i}(t),
$$

where $x_{i}(t), w_{i}(t), u_{i}(t) \in \mathbb{R}^{n}$ are the state, the local coupling, and the control input of the $i$ th node, respectively; $C=\operatorname{diag}\left\{c_{1}, c_{2}, \ldots, c_{n}\right\}, \quad B=\left[b_{k j}\right]_{n \times n} ; \quad F(z(t))=\left[F_{1}\left(z_{1}\right.\right.$ $\left.(t)), F_{2}\left(z_{2}(t)\right), \ldots, F_{n}\left(z_{n}(t)\right)\right]$; and $\alpha$ is the coupling strength. The nonlinear function $F_{k}(t)(k \in[1: n])$ is supposed to satisfy the following Lipschitz condition:

$$
\left|F_{k}(a)-F_{k}(b)\right|^{2} \leq L_{k}|a-b|^{2}, \quad \forall a, b \in \mathbb{R},
$$

where $L_{k}$ is the Lipschitz constant of function $F_{k}$.

2.1. Multiple Partial Coupling. When complex networks are connected via a graph, each node of the network can communicate with its neighboring nodes. Thus, the local coupling of the $i$ th node can be constructed as

$$
w_{i}(t)=\sum_{j=1, j \neq i}^{N} g_{i j}\left(x_{j}(t)-x_{i}(t)\right),
$$

where $N$ is the number of nodes of the network; $G=\left[g_{i j}\right]_{N \times N}$ is the Laplacian matrix representing the 
structural topology of the complex network (1), where $g_{i j}$ is defined as follows: if the $j$ th node can send information to the $i$ th node, $g_{i j}>0$; otherwise, $g_{i j}=0$. Furthermore, the diagonal elements $g_{i i}=-\sum_{j=1, j \neq i}^{N} g_{i j}$. It implies that the complex network would be decoupled when the complex network realizes synchronization.

The local coupling (3) assumes that the node $x_{i}$ can receive all levels of information of the $j$ th node, that is, $x_{j k}$ $(\forall k \in[1: n])$. However, it is a subtle assumption for most biological systems. A more realistic situation is that only part of $x_{j k}$ can transmit information to other nodes. As a result, only partial information of each node can be received. Additionally, for different nodes, the transmitting information is distinct. Note that many real networks have multiweighted connections. For example, in neural networks, neurons send both electrical signals and chemical signals; in supply chain networks, the capital chain, logistic chain, and information chain have separate routes. Thus, the couplings of these kinds of complex networks should be multiple layers to transmit distinct types of signals or chains. To study the multiple and partial features of couplings, the coupling term (3) is transformed as

$$
w_{i}(t)=\sum_{q=1}^{Q} \sum_{j=1, j \neq i}^{N} g_{i j} A_{i j}^{q}\left(x_{j}(t)-x_{i}(t)\right),
$$

where $Q$ is the number of multiple couplings of the complex network (1): $A_{i j}^{q}=\operatorname{diag}\left\{a_{i j}^{q 1}, a_{i j}^{q 2}, \ldots, a_{i j}^{q n}\right\}$ with $a_{i j}^{q k}=0$ or 1 . When $a_{i j}^{q k}=0$, it indicates that the $k$ th level of information of the $i$ th node $\left(x_{i k}\right)$ cannot receive information of the corresponding level of the $j$ th nodes $\left(x_{j k}\right)$ from the $q$ th layer of the coupling. Otherwise, it means that $x_{i k}$ can be updated according to the received information $x_{j k}$.

2.2. Event-Triggered Communication. Until now, each node of the network can send real-time information to its neighboring nodes. However, such a manner of communication will consume more resources on communication media. In particular, it is needless. Recently, the eventtriggered sampling scheme has been introduced as a more efficient substitute. Many existing works designed the event detector of each node. In this work, connections are split into $n$ channels to transmit the corresponding level of node information. The event detector should be installed for each channel. It is utilized to determine the sampling instant of the channel. Specifically, the event condition for $x_{i k}$ is designed as follows:

$$
\left|x_{i k}\left(T_{m}^{i k}\right)-x_{i k}\left(T_{m}^{i k}+l h\right)\right| \leq \gamma_{i k}\left|x_{i k}\left(T_{m}^{i k}+l h\right)-s_{k}\left(T_{m}^{i k}+l h\right)\right|,
$$

where $l \in \mathbb{N}, \gamma_{i k}>0 ; h$ is a positive constant, which denotes the sampling period; $T_{m}^{i k}$ with $m \in \mathbb{N}$ is the latest sampling instant of $x_{i k}$; and $s_{k}(t)$ is the synchronous state, which will be defined later. Thus, the next sampling instant is the time when the event condition (5) is invalid; that is,

$$
T_{m+1}^{i k}=T_{m}^{i k}+h \cdot \min \left\{l \in \mathbb{N}:\left|x_{i k}\left(T_{m}^{i k}\right)-x_{i k}\left(T_{m}^{i k}+l h\right)\right|>\gamma_{i k}\left|x_{i k}\left(T_{m}^{i k}+l h\right)-s_{k}\left(T_{m}^{i k}+l h\right)\right|\right\}
$$

Let the first sampling instant of any $x_{i k}$ be 0 ; that is, $T_{0}^{i k}=$ 0 for all $i \in[1: N]$ and $k \in[1: n]$. Note that the event condition (5) just uses the local information of $x_{i k}$. Thus, it can be conveniently deployed in distributed systems.

Remark 1. The event condition (5) is designed to determine the sequence of sampling instants. When the condition is violated, it means that the difference between the current state and the last sampled state is quite large. Thus, the sampled data is out of date and needs to be refreshed via new sampling. Such a mechanism makes the event-triggered sampling scheme self-adjusting and flexible. The parameter $\gamma_{i k}$ regulates the sampling frequency. When $\gamma_{i k}$ is increasing, it becomes much harder to violate the condition, and fewer samplings will be carried out. However, it also means that less information can be used. One aim of this paper is to choose $\gamma_{i k}$ as large as possible to realize the synchronization of the complex network.

Considering the event-triggering update protocol, the information of any node is invariant during its two contiguous triggered instants. The coupling term of $x_{i k}$ (4) becomes

$$
w_{i k}(t)=\sum_{q=1}^{Q} \sum_{j=1, j \neq i}^{N} g_{i j} a_{i j}^{q k}\left(x_{j k}\left(T_{m(t)}^{j k}\right)-x_{i k}\left(T_{m(t)}^{i k}\right)\right),
$$

where $T_{m(t)}^{i k}\left(T_{m(t)}^{j k}\right)$ is the latest triggered instant of $x_{i k}\left(x_{j k}\right)$ before time $t$. That is,

$$
T_{m(t)}^{i k}=\max \left\{T_{m}^{i k} \leq t: m \in \mathbb{N}\right\}
$$

In this work, the complex network (1) is supposed to synchronize to a given synchronous state $s(t)=\left[s_{1}(t), s_{2}\right.$ $\left.(t), \ldots, s_{n}(t)\right]^{\top} \in \mathbb{R}^{n}$, which satisfies the dynamics of a single node with a given initial state $s(0)$; that is,

$$
\dot{s}(t)=\mathrm{Cs}(t)+\mathrm{BF}(s(t)) \text {. }
$$

Define an error system as $y_{i}(t)=x_{i}(t)-s(t)$ $(i \in[1: N])$. Let

$$
e_{i k}(t)=y_{i k}\left(T_{m(t)}^{i k}\right)-y_{i k}(t), \quad k \in[1: n] .
$$

For $\quad t \in\left[T_{m}^{i k}+l h, T_{m}^{i k}+l h+h\right) \quad(l \geq 0, \quad m \geq 0 \quad$ and $\left.T_{m}^{i k}+l h<T_{m+1}^{i k}\right)$, it can be obtained that 


$$
\begin{aligned}
& x_{j k}\left(T_{m(t)}^{j k}\right)-x_{i k}\left(T_{m(t)}^{i k}\right) \\
& =x_{j k}\left(T_{m\left(T_{m}^{i k}+l h\right)}^{j k}\right)-s_{k}^{*}\left(T_{m\left(T_{m}^{i k}+l h\right)}^{j k}\right)-x_{i k}\left(T_{m}^{i k}\right)+s_{k}^{*}\left(T_{m\left(T_{m}^{i k}+l h\right)}^{j k}\right) \\
& =y_{j k}\left(T_{m\left(T_{m}^{i k}+l h\right)}^{j k}\right)-y_{j k}\left(T_{m}^{i k}+l h\right)-y_{i k}\left(T_{m}^{i k}\right)+y_{i k}\left(T_{m}^{i k}+l h\right)+y_{j k}\left(T_{m}^{i k}+l h\right)-y_{i k}\left(T_{m}^{i k}+l h\right) \\
& =e_{j k}\left(T_{m}^{i k}+l h\right)-e_{i k}\left(T_{m}^{i k}+l h\right)+y_{j k}\left(T_{m}^{i k}+l h\right)-y_{i k}\left(T_{m}^{i k}+l h\right),
\end{aligned}
$$

Based on the above discussions, it can be concluded that

$$
x_{j k}\left(T_{m(t)}^{j k}\right)-x_{i k}\left(T_{m(t)}^{i k}\right)=e_{j k}(m h)-e_{i k}(m h)+y_{j k}(m h)-y_{i k}(m h)
$$

holds for $t \in[m h, m h+h)(m \in \mathbb{N})$. Thus, the coupling term (4) with an event-triggering protocol (12) can be converted as

$$
w_{i}(t)=\sum_{q=1}^{Q} \sum_{j=1, j \neq i}^{N} g_{i j} A_{i j}^{q}\left(e_{j}(m h)-e_{i}(m h)+y_{j}(m h)-y_{i}(m h)\right)
$$

2.3. Pinning Control. To synchronize the complex network (1) to the state $s(t)$, the pinning controllers $u_{i}(t)$ will be deployed. Since the complex network is a large-scale system, it is impossible and unnecessary to control every $x_{i k}$. Controlling a small part of them is sufficient to stabilize the whole network. Specifically, the pinning controller $u_{i k}(t)$ for $x_{i k}$ can be designed as follows:

$$
u_{i k}(t)=d_{i k} \cdot\left(s_{k}\left(T_{m(t)}^{i k}\right)-x_{i k}\left(T_{m(t)}^{i k}\right)\right)
$$

where $d_{i k} \geq 0$ represents the control gain. When $d_{i k}=0, x_{i k}$ is not controlled. It can be seen from (14) that the controller can only use the sampled information.

For $t \in\left[T_{m}^{i k}+l h, T_{m}^{i k}+l h+h\right) \quad(l \geq 0, \quad m \geq 0, \quad$ and $\left.T_{m}^{i k}+l h<T_{m+1}^{i k}\right)$, we can have

$$
\begin{aligned}
u_{i k}(t) & =d_{i k}\left(s_{k}\left(T_{m}^{i k}\right)-x_{i k}\left(T_{m}^{i k}\right)\right) \\
& =d_{i k}\left(-y_{i k}\left(T_{m}^{i k}\right)+y_{i k}\left(T_{m}^{i k}+l h\right)-y_{i k}\left(T_{m}^{i k}+l h\right)\right) \\
& =-d_{i k}\left(e_{i k}\left(T_{m}^{i k}+l h\right)+y_{i k}\left(T_{m}^{i k}+l h\right)\right) .
\end{aligned}
$$

Based on (15), the pinning controller (14) can be transformed to the form of a vector:

$$
u_{i}=-D_{i}\left(e_{i}(m h)+y_{i}(m h)\right)
$$

where $t \in[m h, m h+h)$ and $D_{i}=\operatorname{diag}\left\{d_{i 1}, d_{i 2}, \ldots, d_{i n}\right\}$.

2.4. Complex Networks with Multiple Partial and EventTriggered Couplings. Let $\quad f\left(y_{i}(t)\right)=F\left(x_{i}(t)\right)-$ $F(s(t)) \triangleq\left[f_{1}\left(y_{i 1}(t)\right), f_{1}\left(y_{i 2}(t)\right), \ldots, f_{n}\left(y_{i n}(t)\right)\right]^{\top}$, where $f_{k}\left(y_{i k}(t)\right)=F_{k}\left(x_{i k}(t)\right)-F_{k}\left(s_{k}(t)\right)$, for $\forall k \in[1: n]$. The dynamics of the error system $y_{i}(t)$ can be obtained from (1):

$$
\dot{y}_{i}(t)=C y_{i}(t)+B f\left(y_{i}(t)\right)+\alpha w_{i}(t)+u_{i}(t)
$$

Define matrices $\quad H_{i j}^{q}=\operatorname{diag}\left\{h_{i j}^{q 1}, h_{i j}^{q 2}, \ldots, h_{i j}^{q n}\right\}$ $(q \in[1: Q], i, j \in[1: N])$ as

$$
h_{i j}^{q k}= \begin{cases}g_{i j} a_{i j}^{q k}, & i \neq j, \\ -\sum_{j=1, j \neq i}^{n} g_{i j} a_{i j}^{q k}-d_{i}^{k}, & i=j .\end{cases}
$$

Substituting (13) and (16) into (17), the complex network with multiple partial and event-triggered couplings can be described as

$$
\dot{y}_{i}(t)=C y_{i}(t)+B f\left(y_{i}(t)\right)+\alpha \sum_{q=1}^{Q} \sum_{j=1}^{N} H_{i j}^{q}\left(y_{j}(m h)+e_{j}(m h)\right), \quad i \in[1: N],
$$

where $t \in[m h, m h+h)$ and $m \in \mathbb{N}$. 
The following definition will be needed for the derivation of our main results.

Definition 1. The complex network (1) with multiple partial and event-triggered couplings (13) and the pinning controllers (16) is said to be globally exponential synchronization if, for any initial condition $x_{i}(0)$, there exist positive constants $M$ and $\epsilon$ such that the error system (19) satisfies $\left\|y_{i}(t)\right\| \leq M e^{-\varepsilon t}$ for all $i \in[1: N]$.

The error system (19) is just built for investigating couplings. In this manuscript, the couplings are split into channels. Thus, we should construct a new framework of error system for channels. From (19), it yields that, for $\forall k \in[1: n]$,

$$
\dot{y}_{i k}=c_{k} y_{i k}(t)+\sum_{j=1}^{n} b_{k j} f_{j}\left(y_{i j}(t)\right)+\alpha \sum_{q=1}^{Q} \sum_{j=1}^{N} h_{i j}^{q k}\left(y_{j k}(m h)+e_{j k}(m h)\right) \text {. }
$$

Let $\delta y_{k}(t)=\left[y_{1 k}(t), y_{2 k}(t), \ldots, y_{N k}(t)\right]^{\top}$. Thus, it follows from (20) that

$$
\dot{\delta y_{k}}=c_{k} \delta y_{k}(t)+\sum_{j=1}^{n} b_{k j} \mathbf{f}_{j}\left(\delta y_{j}(t)\right)+\alpha \mathscr{H}_{k}\left[\delta y_{k}(m h)+\delta e_{k}(m h)\right]
$$

where $\quad \mathbf{f}_{j}\left(\delta y_{j}(t)\right)=\left[f_{j}\left(y_{1 j}(t)\right), \ldots, f_{j}\left(y_{N j}(t)\right)\right]^{\top} \quad$ and $\delta e_{k}(m h)=\left[e_{1 k}(m h), \ldots, e_{N k}(m h)\right]^{\top}$.

The error system (21) rearranges channels according to their positions at each coupling, instead of the couplings they belonged to like in (19).

\section{Main Results}

The synchronization criteria will be given for complex network (1) in this section. By introducing a transformation of sampled data, a delay-dependent Lyapunov functional will be built in Theorem 1, which is helpful to reduce the conservatism of our theoretical results.
Theorem 1. Let $\mathscr{H}_{k}=\left[\sum_{q=1}^{Q} h_{i j}^{q k}\right]_{N \times N}(k \in[1: n])$. Under the event-triggered communication condition (5), the complex networks (1) with multiple partial and event-triggered couplings (19) and the pinning controllers (16) can be exponentially synchronized, if there exist positive scalars $\varepsilon_{k}, \varepsilon_{1 k}, \varepsilon_{2 k}$ and matrices $P_{k}>0, \mathscr{W}_{k}=\left[\begin{array}{cc}W_{1 k} & W_{2 k} \\ * & W_{3 k}\end{array}\right]>0, U_{1 k}, U_{2 k}, U_{3 k}$, $U_{4 k}, V_{1 k}, V_{2 k}, X_{k}, X_{1 k}, k \in[1: n]$, such that the following linear matrix inequalities (LMIs) are satisfied:

$$
\begin{gathered}
{\left[\begin{array}{crr}
P_{k}+h \frac{X_{k}+X_{k}^{\top}}{2}+W_{1 k} & -h X_{k}+h X_{1 k}-W_{1 k} & W_{2 k} \\
* & -h X_{1 k}-h X_{1 k}^{\top}+h \frac{X_{k}+X_{k}^{\top}}{2}+W_{1 k} & -W_{2 k} \\
* & * & W_{3 k}
\end{array}\right]>0,} \\
{\left[\begin{array}{cc}
\Omega_{1 k} & \Omega_{2 k} \\
* & \Omega_{3 k}
\end{array}\right]<0,} \\
{\left[\begin{array}{cc}
\widetilde{\Omega}_{1 k} & \widetilde{\Omega}_{2 k} \\
* & \Omega_{3 k}
\end{array}\right]<0,}
\end{gathered}
$$

where 


$$
\begin{aligned}
& \Omega_{1 k}=\left[\begin{array}{ccccc}
\Phi_{11}^{k} & \Phi_{12}^{k} & \Phi_{13}^{k} & \Phi_{14}^{k} & h U_{1 k}^{\top} \\
* & \Phi_{22}^{k} & \Phi_{23}^{k} & \Phi_{24}^{k} & h U_{2 k}^{\top} \\
* & * & \Phi_{33}^{k} & \Phi_{34}^{k} & h U_{3 k}^{\top} \\
* & * & * & \Phi_{44}^{k} & h U_{4 k}^{\top} \\
* & * & * & * & -h W_{1 k}
\end{array}\right], \Omega_{2 k}=\left[\begin{array}{c}
\widetilde{\Omega}_{2 k} \\
0
\end{array}\right], \\
& \widetilde{\Omega}_{1 k}=\left[\begin{array}{cccc}
\Phi_{11}^{k} & \Phi_{12}^{k} & \Pi_{13}^{k} & \Phi_{14}^{k} \\
* & \Pi_{22}^{k} & \Pi_{23}^{k} & \Phi_{24}^{k} \\
* & * & \Pi_{33}^{k} & \Phi_{34}^{k} \\
* & * & * & \Phi_{44}^{k}
\end{array}\right], \widetilde{\Omega}_{2 k}=\left[\begin{array}{cc}
n V_{1 k}^{\top} & 0 \\
0 & 0 \\
0 & n V_{2 k}^{\top} \\
0 & 0
\end{array}\right], \\
& \Omega_{3 k}=\left[\begin{array}{cc}
-n \varepsilon_{1 k} I_{N} & 0 \\
* & -n \varepsilon_{2 k} I_{N}
\end{array}\right] \\
& \Phi_{11}^{k}=-\frac{X_{k}+X_{k}^{\top}}{2}-U_{1 k}-U_{1 k}^{\top}+c_{k} V_{1 k}+c_{k} V_{1 k}^{\top}+\sum_{j=1}^{n}\left(\varepsilon_{1 j}+\varepsilon_{2 j}\right) b_{j k}^{2} L_{k}, \\
& \Phi_{12}^{k}=X_{k}-X_{1 k}-W_{2 k}+U_{1 k}^{\top}-U_{2 k}+\alpha V_{1 k}^{\top} \mathscr{H}_{k} \\
& \Phi_{13}^{k}=P_{k}-U_{3 k}-V_{1 k}^{\top}+c_{k} V_{2 k}, \Phi_{14}^{k}=-U_{4 k}+\alpha V_{1 k}^{\top} \mathscr{H}_{k} \\
& \Phi_{22}^{k}=X_{1 k}+X_{1 k}^{\top}-\frac{X_{k}+X_{k}^{\top}}{2}+W_{2 k}+W_{2 k}^{\top}+U_{2 k}+U_{2 k}^{\top}+\varepsilon_{k} \Gamma_{k}^{2}-h W_{3 k}, \\
& \Phi_{23}^{k}=U_{3 k}+\alpha \mathscr{H}_{k}^{\top} V_{2 k}, \Phi_{24}^{k}=U_{4 k}, \Phi_{33}^{k}=-V_{2 k}-V_{2 k}^{\top}, \Phi_{34}^{k}=\alpha V_{2 k}^{\top} \mathscr{H}_{k}, \\
& \Phi_{44}^{k}=-\varepsilon_{k} I_{N}, \Pi_{13}^{k}=h \frac{X_{k}+X_{k}^{\top}}{2}+P_{k}-U_{3 k}-V_{1 k}^{\top}+c_{k} V_{2 k}, \\
& \Pi_{22}^{k}=X_{1 k}+X_{1 k}^{\top}-\frac{X_{k}+X_{k}^{\top}}{2}+W_{2 k}+W_{2 k}^{\top}+U_{2 k}+U_{2 k}^{\top}+\varepsilon_{k} \Gamma_{k}^{2}+h W_{3 k}, \\
& \Pi_{23}^{k}=U_{3 k}+\alpha \mathscr{H}_{k}^{\top} V_{2 k}+h\left(W_{2 k}^{\top}+X_{1 k}^{\top}-X_{k}^{\top}\right), \Pi_{33}^{k}=-V_{2 k}-V_{2 k}^{\top}+h W_{1 k} \text {. }
\end{aligned}
$$

Proof. Thanks to the Schur complement [31], it follows from (23) and (24) that 


$$
\begin{aligned}
\Psi_{k}= & {\left[\begin{array}{cccc}
\Psi_{11}^{k} & \Phi_{12}^{k} & \Phi_{13}^{k} & h U_{1 k}^{\top} \\
* & \Phi_{22}^{k} & \Phi_{23}^{k} & h U_{2 k}^{\top} \\
* & * & \Psi_{33}^{k} & h U_{3 k}^{\top} \\
* & * & * & -h W_{1 k}
\end{array}\right]<0, } \\
\Psi_{k}^{\prime} & =\left[\begin{array}{cccc}
\Psi_{11}^{k} & \Phi_{12}^{k} & \Pi_{13}^{k} & \Phi_{14}^{k} \\
* & \Pi_{22}^{k} & \Phi_{23}^{k} & \Phi_{24}^{k} \\
* & * & \Psi_{33}^{k} & \Pi_{34}^{k} \\
* & * & * & \Pi_{44}^{k}
\end{array}\right]<0
\end{aligned}
$$

where $\quad \Psi_{11}^{k}=\Phi_{11}^{k}+n \varepsilon_{1 k}^{-1} V_{1 k}^{\top} V_{1 k}, \quad \Psi_{33}^{k}=\Phi_{33}^{k}+n \varepsilon_{2 k}^{-1} V_{2 k}^{\top} V_{2 k}$, and $\Psi_{33}^{k}=\Pi_{33}^{k}+n \varepsilon_{2 k}^{-1} V_{2 k}^{\top} V_{2 k}$.

Basing on the error system (21), we can establish the Lyapunov functional $V(t)=\sum_{k=1}^{n} V_{k}(t)$, where

$$
V_{k}(t)=\delta y_{k}^{\top}(t) P_{k} \delta y_{k}(t)+\varsigma(t) \int_{m h}^{t}\left[\begin{array}{c}
\delta y_{k}(s) \\
\delta y_{k}(m h)
\end{array}\right]^{\top} \mathscr{W}_{k}\left[\begin{array}{c}
\delta y_{k}(s) \\
\delta y_{k}(m h)
\end{array}\right] \mathrm{d} s+\varsigma(t)\left[\begin{array}{c}
\delta y_{k}(t) \\
\delta y_{k}(m h)
\end{array}\right]^{\top} x_{k}\left[\begin{array}{c}
\delta y_{k}(t) \\
\left(\delta y_{k}(m h)\right.
\end{array}\right], \quad t \in[m h,(m+1) h]
$$

$\zeta(t)=(m+1) h-t \quad$ and
$\left[\begin{array}{cc}X_{k}+X_{k}^{\top} / 2 & -X_{k}+X_{1 k} \\ * & -X_{1 k}-X_{1 k}^{\top}+X_{k}+X_{k}^{\top} / 2\end{array}\right] ; \delta y_{k}(t)$ is defined in (21).

The rest of the proof consists of three steps. The first step will indicate the Lyapunov functional $V(t)$ is well defined, that is, $V(t) \geq 0$, and $V(t)=0$ if and only if $\delta y_{k}(t)=0$ holds for all $k \in[1: n]$. The second step will show that $V(t)$ is exponential convergent under conditions (22)-(24). Finally, the error system is exponential stable (19), and the complex network (1) can reach synchronization according to Theorem 1.

Step 1. Well-definedness of $V(t)$.

Considering $\mathscr{W}_{k}>0$ and the Schur complement [31], it follows that $\tilde{W}_{k} \triangleq W_{1 k}-W_{2 k} W_{3 k}^{-1} W_{2 k}^{\top}>0$. Thus, it follows that

$$
\mathscr{W}_{k}-\left[\begin{array}{cc}
\widehat{W}_{k} & 0 \\
* & 0
\end{array}\right]=\left[\begin{array}{cc}
W_{2 k} W_{3 k}^{-1} W_{2 k}^{\top} & W_{2 k} \\
* & W_{3 k}
\end{array}\right] \geq 0
$$

which further implies

$$
\mathscr{W}_{k} \geq\left[\begin{array}{cc}
\widehat{W}_{k} & 0 \\
* & 0
\end{array}\right]
$$

Based on the above inequality, it yields that

$$
\left[\begin{array}{c}
\delta y_{k}(s) \\
\delta y_{k}(m h)
\end{array}\right]^{\top} \mathscr{W}_{k}\left[\begin{array}{c}
\delta y_{k}(s) \\
\delta y_{k}(m h)
\end{array}\right] \geq \dot{\delta} y_{k}^{\top}(s) \widehat{W}_{k} \dot{\delta} y_{k}(s)
$$

Thus, it yields from (28) and (31) that

$$
V_{k}(t) \geq \delta y_{k}^{\top}(t) P_{k} \delta y_{k}(t)+\varsigma(t) \int_{m h}^{t} \dot{\delta} y_{k}^{\top}(s) \widehat{W}_{k} \dot{\delta} y_{k}(s) \mathrm{d} s+\varsigma(t)\left[\begin{array}{c}
\delta y_{k}(t) \\
\delta y_{k}(m h)
\end{array}\right]^{\top} \mathscr{X}_{k}\left[\begin{array}{c}
\delta y_{k}(t) \\
\delta y_{k}(m h)
\end{array}\right]
$$

Resorting to the Jensen inequality [32], it can be obtained that

$$
\begin{aligned}
& \int_{m h}^{t} \dot{\delta y_{k}^{\top}}(s) \widehat{W}_{k} \dot{\delta} y_{k}(s) \mathrm{d} s \geq \frac{1}{\tau(t)} \int_{m h}^{t} \dot{\delta} y_{k}^{\top}(s) \mathrm{d} s \widehat{W}_{k} \int_{m h}^{t} \dot{\delta} y_{k}(s) \mathrm{d} s \\
& \geq \frac{1}{h}\left[\delta y_{k}(t)-\delta y_{k}(m h)\right]^{\top} \widehat{W}_{k}\left[\delta y_{k}(t)-\delta y_{k}(m h)\right],
\end{aligned}
$$

where $\tau(t)=t-m h$. It follows from (32) and (33) that 


$$
V_{k}(t) \geq\left[\begin{array}{c}
\delta y_{k}(t) \\
\delta y_{k}(m h)
\end{array}\right]^{\top}\left[\frac{\varsigma(t)}{h}\left(P_{k}+h \mathscr{X}_{k}+\widehat{\mathscr{W}}_{k}\right)+\frac{\tau(t)}{h} P_{k}\right]\left[\begin{array}{c}
\delta y_{k}(t) \\
\delta y_{k}(m h)
\end{array}\right],
$$

where $P_{k}=\left[\begin{array}{cc}P_{k} & 0 \\ * & 0\end{array}\right]$ and $\widehat{\mathscr{W}}_{k}=\left[\begin{array}{cc}\widehat{W}_{k} & -\widehat{W}_{k} \\ * & \widehat{W}_{k}\end{array}\right]$. By applying the Schur complement [31], it can be obtained from (22) that $P_{k}+h X_{k}+\widetilde{\mathscr{W}}_{k}>0$. Hence, $V_{k}(t)$ defined in (28) is well defined. Furthermore, there must exist a number $\beta \in(0,1)$ such that $P_{k}+h \mathscr{X}_{k}+\widehat{\mathscr{W}}_{k}>\beta P_{k}$. It follows from (34) that

$$
V_{k}(t)>\beta\left[\begin{array}{c}
\delta y_{k}(t) \\
\delta y_{k}(m h)
\end{array}\right]^{\top} P_{k}\left[\begin{array}{c}
\delta y_{k}(t) \\
\delta y_{k}(m h)
\end{array}\right]=\beta \delta y_{k}^{\top}(t) P_{k} \delta y_{k}(t) .
$$

Due to $P_{k}>0, V_{k}(t) \geq 0$ and the equality holds if and only if $\operatorname{deltay}_{k}(t)=0$. Thus, the Lyapunov functional $V(t)$ is well defined.
Step 2. Exponential convergence of $V(t)$

The derivative of $V_{k}(t)$ is

$$
\begin{aligned}
\dot{V}_{k}(t)= & 2 \dot{\delta} y_{k}(t) P_{k} \delta y_{k}(t) \\
& -\int_{m h}^{t}\left[\begin{array}{c}
\dot{\delta} y_{k}(s) \\
\delta y_{k}(m h)
\end{array}\right]^{\top} \mathscr{W}_{k}\left[\begin{array}{c}
\dot{\delta} y_{k}(s) \\
\delta y_{k}(m h)
\end{array}\right] \mathrm{d} s+\varsigma(t)\left[\begin{array}{c}
\delta y_{k}(t) \\
\delta y_{k}(m h)
\end{array}\right]^{\top} \mathscr{W}_{k}\left[\begin{array}{c}
\dot{\delta} y_{k}(t) \\
\delta y_{k}(m h)
\end{array}\right] \\
& -\left[\begin{array}{c}
\delta y_{k}(t) \\
\delta y_{k}(m h)
\end{array}\right]^{\top} \mathscr{X}_{k}\left[\begin{array}{c}
\delta y_{k}(t) \\
\delta y_{k}(m h)
\end{array}\right]+2 \varsigma(t)\left[\begin{array}{c}
\delta y_{k}(t) \\
0
\end{array}\right]^{\top} \mathscr{X}_{k}\left[\begin{array}{c}
\delta y_{k}(t) \\
\delta y_{k}(m h)
\end{array}\right] .
\end{aligned}
$$

Thanks to the Jensen inequality [32], it can be obtained

that

$$
\begin{aligned}
& \int_{m h}^{t}\left[\begin{array}{c}
\delta y_{k}(s) \\
\delta y_{k}(m h)
\end{array}\right]^{\top} \mathscr{W}_{k}\left[\begin{array}{c}
\delta y_{k}(s) \\
\delta y_{k}(m h)
\end{array}\right] \mathrm{d} s \\
& \geq \tau(t) \phi_{k}^{\top}(t) W_{1 k} \phi_{k}(t)+2\left[\delta y_{k}(t)-\delta y_{k}(m h)\right]^{\top} W_{2 k} \delta y_{k}(m h)+\tau(t) \delta y_{k}^{\top}(m h) W_{3 k} \delta y_{k}(m h),
\end{aligned}
$$

where $\phi(t)=1 / \tau(t) \int_{m h}^{t} \dot{\delta} y_{k}(s) \mathrm{d} s$. Based on the Newton-Leibnitz formula, we can have

$$
0=\sum_{k=1}^{n} 2\left[\delta y_{k}^{\top}(t) U_{1 k}^{\top}+\delta y_{k}^{\top}(m h) U_{2 k}^{\top}+\delta \dot{y}_{k}^{\top}(t) U_{3 k}^{\top}+\delta e_{k}^{\top}(m h) U_{4 k}^{\top}\right] \cdot\left[-\delta y_{k}(t)+\delta y_{k}(m h)+\tau(t) \phi_{k}(t)\right]
$$

From (21), one has that

$$
0=\sum_{k=1}^{n} 2\left[\delta y_{k}^{\top}(t) V_{1 k}^{\top}+\dot{\delta} y_{k}(t) V_{2 k}^{\top}\right] \cdot\left[-\dot{\delta} y_{k}(t)+c_{k} \delta y_{k}(t)+\sum_{j=1}^{n} b_{k j} \mathbf{f}_{j}\left(\delta y_{j}(t)\right)+\alpha \mathscr{H}_{k}\left(\delta e_{k}(m h)+\delta y_{k}(m h)\right)\right]
$$


Based on the Lipschitz condition (2), we have that

$$
\begin{gathered}
2 \delta y_{k}^{\top}(t) V_{1 k}^{\top} \sum_{j=1}^{n} b_{k j} \mathbf{f}_{j}\left(\delta y_{j}(t)\right) \leq n \varepsilon_{1 k}^{-1} \delta y_{k}^{\top}(t) V_{1 k}^{\top} V_{1 k} \delta y_{k}(t)+\varepsilon_{1 k} \sum_{j=1}^{n} b_{k j}^{2} \delta y_{j}^{\top}(t) L_{j} \delta y_{j}(t) . \\
2 \dot{\delta} y_{k}^{\top}(t) V_{2 k}^{\top} \sum_{j=1}^{n} b_{k j} \mathbf{f}_{j}\left(\delta y_{j}(t)\right) \leq n \varepsilon_{2 k}^{-1} \delta y_{k}^{\top}(t) V_{2 k}^{\top} V_{2 k} \delta y_{k}(t)+\varepsilon_{2 k} \sum_{j=1}^{n} b_{k j}^{2} \delta y_{j}^{\top}(t) L_{j} \delta y_{j}(t) .
\end{gathered}
$$

Considering the event condition (5), we can have that

$$
\begin{aligned}
& \text { LHS }=\left|y_{i k}\left(T_{m}^{i k}\right)-y_{i k}\left(T_{m}^{i k}+l h\right)\right|=\left|e_{i k}\left(T_{m}^{i k}+l h\right)\right|, \\
& \text { RHS }=\gamma_{i k}\left|y_{i k}\left(T_{m}^{i k}+l h\right)\right| .
\end{aligned}
$$

Generally, it yields for any moment $t=m h(m \in \mathbb{N})$ that

$$
\left|e_{i k}(m h)\right| \leq \gamma_{i k}\left|y_{i k}(m h)\right|
$$

holds for any $m \in \mathbb{N}, i \in[1: N]$, and $k \in[1: n]$, which further implies that

$$
\varepsilon_{k}\left(\delta e_{k}(m h)\right)^{\top} \delta e_{k}(m h) \leq \varepsilon_{k}\left(\delta y_{k}(m h)\right)^{\top} \Gamma_{k}^{2} \delta y_{k}(m h),
$$

where $\Gamma_{k}=\operatorname{diag}\left\{\gamma_{1 k}, \gamma_{2 k}, \ldots, \gamma_{N k}\right\}$ and $\varepsilon_{k}$ can be any positive number.

Combining (36)-(44), we have that

$$
\dot{V}(t) \leq \sum_{k=1}^{n} \eta_{k}^{\top}(t)\left[\frac{\varsigma(t)}{h} \bar{\Psi}_{k}+\frac{\tau(t)}{h} \Psi_{k}\right] \eta_{k}(t),
$$

where $\eta_{k}(t)=\left[\delta y_{k}(t) \delta y_{k}(m h) \dot{\delta} y_{k}(t) \delta e(m h) \phi_{k}(t)\right]^{\top}$ and $\bar{\Psi}_{k}=\left[\begin{array}{cc}\Psi_{k}^{\prime} & 0 \\ * & 0\end{array}\right]$. From (26) and (27), one can always find a sufficiently small positive number $\varepsilon$ such that $\dot{V}(t)+\varepsilon V(t)<0$ holds for $\forall t \in[m h,(m+1) h)$. Solving the differential equation, we have $V(t)<e^{-\varepsilon(t-m h)} V(m h)$. Similarly, we can get $V(m h)<e^{-\varepsilon h} V(m h-h)$ for any $m \in \mathbb{N}$. Combining all these inequalities yields that $V(t)<e^{-\varepsilon t} V(0), \forall t>0$. Thus, the Lyapunov functional $V(t)$ is exponential convergence.

Step 3. Exponential synchronization of complex network (1).

Considering the error system (19), we have that

$$
\begin{aligned}
\left\|y_{i}(t)\right\|^{2} & \leq \sum_{i=1}^{N} y_{i}^{\top}(t) y_{i}(t) \\
& =\sum_{k=1}^{n} \delta y_{k}^{\top}(t) \delta y_{k}(t) \\
& \leq \sum_{k=1}^{n} \lambda_{\min }^{-1}\left(P_{k}\right) \delta y_{k}^{\top}(t) P_{k} \delta y_{k}(t) \\
& <\beta^{-1} \max _{k=1}^{n} \lambda_{\min }^{-1}\left(P_{k}\right) V(t) \\
& <\beta^{-1} \max _{k=1}^{n} \lambda_{\min }^{-1}\left(P_{k}\right) V(0) e^{-\epsilon t} .
\end{aligned}
$$

Based on Definition 1, the complex network (1) with multiple partial and event-triggered couplings (13) and the pinning controllers (16) is exponential synchronization. This completes the proof.

Remark 2. Most existing results studied the macro-level connections among nodes. In this work, only a part of the channel of a node can communicate. Thus, the micro-level connections among nodes should also be investigated. A feasible method is to build an augmented system to contain both macro and micro connections. However, it will lead to a large-dimension stabilization condition, which is hard to be verified. An alternative way is to rearrange neurons. Equation (21) collects all the channels which have the same index of each node, instead of the channels that belonged to the same node like equation (1). The complex network (13) is rearranged as $n$ subsystems. By establishing the Lyapunov functional (21) for each subsystem, the stabilization condition with smaller dimensions can be obtained.

The time-dependent Lyapunov functional is constructed in Theorem 1 to deal with the sampled information. This method was introduced in [33] and improved in [34]. These Lyapunov functionals do not grow after the sampling instants. Thus, the conservatism of the main results can be reduced further. To show this fact, the following theorem is derived, in which a time-independent Lyapunov functional is established. 
Theorem 2. The complex networks (1) with multiple partial and event-triggered couplings (13) and the pinning controllers (16) can achieve exponential synchronization if there exist positive scalars $\varepsilon_{k}, \varepsilon_{1 k}, \varepsilon_{2 k}$ and matrices $P_{k}>0, Q_{k}>0, R_{k}>0$, $V_{1 k}, V_{2 k}, S_{1 k}, S_{2 k}, U_{1 k}, U_{2 k}, k \in[1: n]$, such that the following

$$
\left[\begin{array}{cc}
\Omega_{1 k} & \Omega_{2 k} \\
* & \Omega_{3 k}
\end{array}\right]<0
$$

where LMIs are satisfied:

$$
\begin{aligned}
\Omega_{1 k} & =\left[\begin{array}{ccccc}
\Phi_{11}^{k} & \Phi_{12}^{k} & 0 & \Phi_{14}^{k} & \alpha V_{1 k}^{\top} \mathscr{H}_{k} \\
* & \Phi_{22}^{k} & \Phi_{23}^{k} & \Phi_{24}^{k} & 0 \\
* & * & \Phi_{33}^{k} & 0 & 0 \\
* & * & * & \Phi_{44}^{k} & \alpha V_{2 k}^{\top} \mathscr{H}_{k} \\
* & * & * & * & -\varepsilon_{k} I_{N}
\end{array}\right], \Omega_{2 k}=\left[\begin{array}{cccc}
h U_{1 k} & 0 & n V_{1 k}^{\top} & 0 \\
h U_{2 k} & h S_{2 k} & 0 & 0 \\
0 & h S_{1 k} & 0 & 0 \\
0 & 0 & 0 & n V_{2 k}^{\top}
\end{array}\right], \\
\Omega_{3 k} & =\operatorname{diag}\left\{-h R_{k},-h R_{k},-n \varepsilon_{1 k} I_{N},-n \varepsilon_{2 k} I_{N}\right\}, \\
\Phi_{11}^{k} & =Q_{k}+U_{1 k}+U_{1 k}^{\top}+c_{k} V_{1 k}+c_{k} V_{1 k}^{\top}+\sum_{j=1}^{n}\left(\varepsilon_{1 j}+\varepsilon_{2 j}\right) b_{j k}^{2} L_{k}, \\
\Phi_{12}^{k} & =-U_{1 k}+U_{2 k}^{\top}+\alpha V_{1 k}^{\top} \mathscr{H}_{k}, \Phi_{22}^{k}=-U_{2 k}-U_{2 k}^{\top}+S_{2 k}+S_{2 k}^{\top}+\varepsilon_{k} \Gamma_{k}^{2}, \\
\Phi_{23}^{k} & =S_{1 k}^{\top}-S_{2 k}, \Phi_{14}^{k}=P_{k}-V_{1 k}^{\top}+c_{k} V_{2 k}, \\
\Phi_{24}^{k} & =\alpha \mathscr{H}_{k}^{\top} V_{2 k}, \Phi_{33}^{k}=-Q_{k}-S_{1 k}-S_{1 k}^{\top}, \Phi_{44}^{k}=h R_{k}-V_{2 k}-V_{2 k}^{\top} .
\end{aligned}
$$

Proof. Consider the following Lyapunov functional $V(t)=\sum_{k=1}^{n} V_{k}(t)$, where

$$
V_{k}(t)=\delta y_{k}^{\top}(t) P_{k} \delta y_{k}(t)+\int_{t-h}^{t} \delta y_{k}^{\top}(s) Q_{k} \delta y_{k}(s) \mathrm{d} s+\int_{-h}^{0} \int_{t+\theta}^{t} \dot{\delta} y_{k}^{\top}(s) R_{k} \dot{\delta} y_{k}(s) \mathrm{d} s \mathrm{~d} \theta
$$

The derivative of $V_{k}(t)$ is

$$
\dot{V}_{k}(t)=2 \dot{\delta} y_{k}^{\top}(t) P_{k} \delta y_{k}(t)+\delta y_{k}^{\top}(t) Q_{k} \delta y_{k}(t)-\delta y_{k}^{\top}(t-h) Q_{k} \delta y_{k}(t-h)+h \dot{\delta} y_{k}^{\top}(t) R_{k} \dot{\delta} y_{k}(t)-\int_{t-h}^{t} \dot{\delta y_{k}^{\top}}(\theta) R_{k} \dot{\delta} y_{k}(\theta) \mathrm{d} \theta
$$

The Newton-Leibniz formula gives that

$$
\begin{gathered}
2\left[\delta y_{k}^{\top}(t) U_{1 k}+\delta y_{k}^{\top}(m h) U_{2 k}\right] \cdot\left[\delta y_{k}(t)-\delta y_{k}(m h)-\int_{m h}^{t} \delta y_{k}(s) \mathrm{d} s\right]=0, \\
2\left[\delta y_{k}^{\top}(t) S_{1 k}+\delta y_{k}^{\top}(m h) S_{2 k}\right] \cdot\left[\delta y_{k}(m h)-\delta y_{k}(t-h)-\int_{t-h}^{m h} \delta y_{k}(s) \mathrm{d} s\right]=0,
\end{gathered}
$$

where $m=\max \{m \in \mathbb{N} \mid m h<t\}$. Adding the left side of (51) and (52) into (50) yields 


$$
\begin{aligned}
\dot{V}_{k}(t)= & 2 \dot{\delta} y_{k}^{\top}(t) P_{k} \delta y_{k}(t)+\delta y_{k}^{\top}(t) Q_{k} \delta y_{k}(t)-\delta y_{k}^{\top}(t-h) Q_{k} \delta y_{k}(t-h)+h \dot{\delta} y_{k}^{\top}(t) R_{k} \dot{\delta} y_{k}(t) \\
& +2\left[\delta y_{k}^{\top}(t) U_{1 k}+\delta y_{k}^{\top}(m h) U_{2 k}\right]\left[\delta y_{k}(t)-\delta y_{k}(m h)\right]-\int_{m h}^{t} \zeta_{k}^{\top}(t, \theta) R_{k}^{-1} \zeta_{k}(t, \theta) \mathrm{d} \theta+2\left[\delta y_{k}^{\top}(t-h) S_{1 k}+\delta y_{k}^{\top}(m h) S_{2 k}\right] \\
& {\left[\delta y_{k}(m h)-\delta y_{k}(t-h)\right] } \\
& -\int_{t-h}^{m h} \eta_{k}^{\top}(t, \theta) R_{k}^{-1} \eta_{k}(t, \theta) \mathrm{d} \theta+(t-m h)\left[\delta y_{k}^{\top}(t) U_{1 k}+\delta y_{k}^{\top}(m h) U_{2 k}\right] R_{k}^{-1}\left[U_{1 k}^{\top} \delta y_{k}(t)+U_{2 k}^{\top} \delta y_{k}(m h)\right]+[m h-(t-h)] \\
& {\left[\delta y_{k}^{\top}(t-h) S_{1 k}+\delta y_{k}^{\top}(m h) S_{2 k}\right] R_{k}^{-1}\left[S_{1 k}^{\top} \delta y_{k}(t-h) S_{2 k}^{\top} \delta y_{k}(m h)\right] } \\
\leq & 2 \dot{\delta} y_{k}^{\top}(t) P_{k} \delta y_{k}(t)+\delta y_{k}^{\top}(t) Q_{k} \delta y_{k}(t)-\delta y_{k}^{\top}(t-h) Q_{k} \delta y_{k}(t-h)+h \delta y_{k}^{\top}(t) R_{k} \delta y_{k}(t)+2\left[\delta y_{k}^{\top}(t) U_{1 k}+\delta y_{k}^{\top}(m h) U_{2 k}\right] \\
& {\left[\delta y_{k}(t)-\delta y_{k}(m h)\right] } \\
& +2\left[\delta y_{k}^{\top}(t-h) S_{1 k}+\delta y_{k}^{\top}(m h) S_{2 k}\right](t-h)+h\left[\delta y_{k}^{\top}(t) U_{1 k}+\delta y_{k}^{\top}(m h) U_{2 k}\right] R_{k}^{-1}\left[U_{1 k}^{\top} \delta y_{k}(t)+U_{2 k}^{\top} \delta y_{k}(m h)\right] \\
& +h\left[\delta y_{k}^{\top}(t-h) S_{1 k}+\delta y_{k}^{\top}(m h) S_{2 k}\right] R_{k}^{-1}\left[S_{1 k}^{\top} \delta y_{k}(t-h)+S_{2 k}^{\top} \delta y_{k}(m h)\right],
\end{aligned}
$$

where $\zeta_{k}(t, \theta)=U_{1 k}^{\top} \delta y_{k}(t)+U_{2 k}^{\top} \delta y_{k}(m h)+R_{k} \dot{\delta} y_{k}(\theta)$ and $\eta_{k}(t, \theta)=S_{1 k}^{\top} \delta y_{k}(t-h)+S_{2 k}^{\top} \delta y_{k}(m h)+R_{k} \delta y_{k}(\theta)$. Considering (39), the Lipschitz assumption of function $f(40)$ and (41), and the event condition (44), it yields that

$$
\dot{V}_{k}(t) \leq \theta_{k}^{\top}(t) \Xi_{k} \theta_{k}(t)
$$

where $\quad \theta_{k}(t)=\left[\delta y_{k}(t) \delta y_{k}(m h) \delta y_{k}(t-h) \delta \dot{y}_{k}(t) \delta e_{k}\right.$ $(m h)]^{\top}$,

$$
\begin{aligned}
\Xi_{k} & =\left[\begin{array}{ccccc}
\Xi_{11}^{k} & \Phi_{12}^{k}+h U_{1 k} R_{k}^{-1} U_{2 k}^{\top} & 0 & \Phi_{14}^{k} & \alpha V_{1 k}^{\top} \mathscr{H}_{k} \\
* & \Xi_{22}^{k} & -S_{2 k}+h S_{2 k} R_{k}^{-1} S_{1 k}^{\top} & \Phi_{24}^{k} & 0 \\
* & * & -Q_{k}+h S_{1 k} R_{k}^{-1} S_{1 k}^{\top} & 0 & 0 \\
* & * & * & \Phi_{44}^{k}+n \varepsilon_{2 k}^{-1} V_{2 k}^{\top} V_{2 k} & \alpha V_{2 k}^{\top} \mathscr{H}_{k} \\
* & * & * & * & -\varepsilon_{k} I_{N}
\end{array}\right], \\
\Xi_{11}^{k} & =\Phi_{11}^{k}+h U_{1 k} R_{k}^{-1} U_{1 k}^{\top}+n \varepsilon_{1 k}^{-1} V_{1 k}^{\top} V_{1 k}, \\
\Xi_{22}^{k} & =\Phi_{22}^{k}+h U_{2 k} R_{k}^{-1} U_{2 k}^{\top}+h S_{2 k} R_{k}^{-1} S_{2 k}^{\top} .
\end{aligned}
$$

Thanks to the Schur complement [31], the negative definiteness of $\Xi_{k}$ can be guaranteed by (47). Thus, $\dot{V}_{k}(t)<0$. By using the same derivation in Theorem 1 , it can be concluded that the complex network (1) with multiple partial and event-triggered couplings (13) and the pinning controllers (16) can realize exponential synchronization according to Definition 1. This completes the proof.

\section{Simulations}

In this section, a simulative example will be built to illustrate the efficiency and effectiveness of the proposed synchronization criteria. In particular, a comparison will be presented to show how much conservatism can be reduced by Theorem 1 .

A complex network with four nodes $(N=4)$ is considered, each of which has three levels of information $(n=3)$. The parameters for each node are $\dot{x}(t)=C x(t)+$ $\mathrm{BF}(x(t))$ with $F_{1}(u)=F_{2}(u)=F_{3}(u)=|u+1|-|u-1| / 2$ and

$$
C=\left[\begin{array}{ccc}
-0.1 & 0 & 0 \\
0 & -0.1 & 0 \\
0 & 0 & -0.1
\end{array}\right], B=\left[\begin{array}{ccc}
0.116 & -0.15 & -0.15 \\
-0.15 & 0.116 & -0.2 \\
-0.12 & 0.2 & 0.116
\end{array}\right], G=\left[\begin{array}{cccc}
-3 & 1 & 1 & 1 \\
1 & -2 & 1 & 0 \\
1 & 1 & -3 & 1 \\
1 & 0 & 1 & -2
\end{array}\right]
$$


The function $F_{k}$ satisfies the Lipschitz condition (2) with the Lipschitz constants $L_{k}=1(k \in[1: 3])$. The coupling strength of the complex network is $\alpha=0.35$; $G$ is the Laplacian matrix of the connections among nodes. The trajectory of the synchronous state $s(t)$ with the initial state $s(0)=[-1,0,1]^{\top}$ is depicted in Figure 1.

For any pair of adjacent nodes, only a part of levels of information can communicate, which leads to partial couplings. Furthermore, the layers of couplings are duplex $(Q=2)$ in this example. Thus, two diagonal matrices are employed for each connection, that is, $g_{i j}>0$. They are listed as follows:

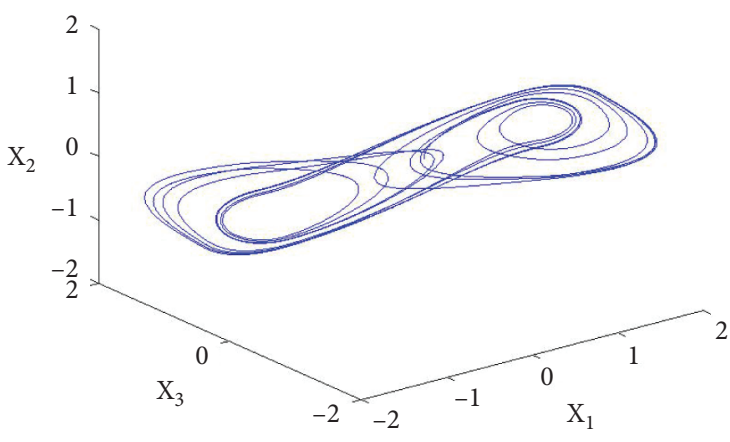

Figure 1: The trajectory of the synchronous state $s(t)$.

$$
\begin{aligned}
& A_{12}^{1}=\operatorname{diag}\{1,0,0\}, A_{12}^{2}=\operatorname{diag}\{1,0,1\}, A_{13}^{1}=\operatorname{diag}\{0,1,0\}, A_{13}^{2}=\operatorname{diag}\{0,0,1\}, \\
& A_{14}^{1}=\operatorname{diag}\{0,1,1\}, A_{14}^{2}=\operatorname{diag}\{0,1,1\}, A_{21}^{1}=\operatorname{diag}\{1,0,1\}, A_{21}^{2}=\operatorname{diag}\{0,0,1\}, \\
& A_{23}^{1}=\operatorname{diag}\{1,0,0\}, A_{23}^{2}=\operatorname{diag}\{1,1,0\}, A_{31}^{1}=\operatorname{diag}\{0,0,0\}, A_{31}^{2}=\operatorname{diag}\{0,0,1\}, \\
& A_{32}^{1}=\operatorname{diag}\{0,0,1\}, A_{32}^{2}=\operatorname{diag}\{1,0,1\}, A_{34}^{1}=\operatorname{diag}\{0,1,1\}, A_{34}^{2}=\operatorname{diag}\{0,0,0\}, \\
& A_{41}^{1}=\operatorname{diag}\{1,0,0\}, A_{41}^{2}=\operatorname{diag}\{1,0,0\}, A_{43}^{1}=\operatorname{diag}\{0,1,1\}, A_{12}^{2}=\operatorname{diag}\{1,0,1\} .
\end{aligned}
$$

For each channel, an event condition (5) should be designed to realize the event-triggered sampling protocol. The sampling period $h$ is set as 0.2 and $\gamma_{i k}=0.17$, for all channels $(i \in[1: 4], k \in[1: 3])$. Furthermore, a portion of $x_{i k}$ deploy the feedback controllers (14). That is, $d_{11}=2$, $d_{22}=2, d_{23}=2, d_{31}=2, d_{32}=2, d_{42}=2$, and $d_{43}=2$.

By using the MATLAB LMI toolbox, the synchronization criteria (22)-(24) have a feasible solution. Based on Theorem 1, the constructed complex network (1) with multiple partial and event-triggered couplings (13) and the pinning controllers (16) can achieve synchronization. To show this fact, a simulation is given. The initial state $x_{i}(0)$ is randomly chosen from the interval $[-5,5]$. Figure 2 displays the trajectories of the error system (19) of the complex network. It can be found that all errors $y_{i}(t)(i \in[1: 4])$ tend to 0 , which means that the synchronization can be realized.

To show the superiority of the event-triggered communication, we compare it with the time-triggered communication. Let $M_{i k}(t)$ be the number of times that the event condition (5) is violated for $x_{i k}$. That is, $M_{i k}(t)=m(t)$, where $m(t)$ is the one in $T_{m(t)}^{i k}$ which is defined in (8). If the complex network samples information for every 0.2 interval, the total sampling number of the whole network at time $t$ would be $12 \times\lfloor t / 0.2\rfloor$, where $\lfloor x\rfloor$ is the floor function, that is, the greatest integer less than or equal to $x$. Let $R(t)$ be ratio between the number of the event-triggered sampling and that of the time-triggered sampling number:

$$
R(t)=\frac{\sum_{i=1}^{4} \sum_{k=1}^{3} M_{i k}(t)}{12 \times\lfloor t / 0.2\rfloor} .
$$

Figure 3 shows the trajectory of $R(t)$ for $t \leq 20$. It can be seen that $R(t)$ decreases rapidly and stabilizes around $53 \%$. It indicates that the event-triggered sampling scheme can save near half of the communication resources. This is a great advantage to its time-triggered counterpart.

Finally, we will compare the conservatism of conditions in Theorems 1 and 2. Assume that all $x_{i k}$ have the same $\gamma$ for the event condition (5), that is, $\gamma_{i k}=\gamma(i \in[1: 4], k \in[1: 3])$. By changing the sampling period $h$, we try to find the maximum $\gamma$ which allows the existence of feasible solutions for two theorems. As discussed in Remark 1, larger $\gamma$ makes the event harder to be triggered. It means that the complex network can be synchronized with less frequent sampling and then has less conservatism. Figure 4 shows the maximum $\gamma s$ which can be found in Theorems 1 and 2. It can be seen that, for any fixed $h$, $\gamma$ for Theorem 1 (the solid curve) is larger than the one for Theorem 2 (the dashed curve), especially when the sampling period $h$ is increasing. Thus, Theorem 1 has less conservatism than Theorem 2. It is the merit of the delay-dependent Lyapunov functional (28).

\section{Conclusion}

The pinning synchronization problem of complex networks with multiple partial and event-triggered couplings has been studied in this paper. An event detector has been designed for every level of information of each node to decide the sampling instants. Furthermore, only a part of channels of connections can communicate with neighboring nodes. Thus, partial information of each node is available. Furthermore, couplings have multiple layers to transmit various kinds of information. To synchronize the complex network, the pinning control method has been used. The control input can only utilize the sampled information. Based on a rearrangement of the channels of couplings, a delay-dependent Lyapunov functional has been constructed. The synchronization criteria have been proposed with less 

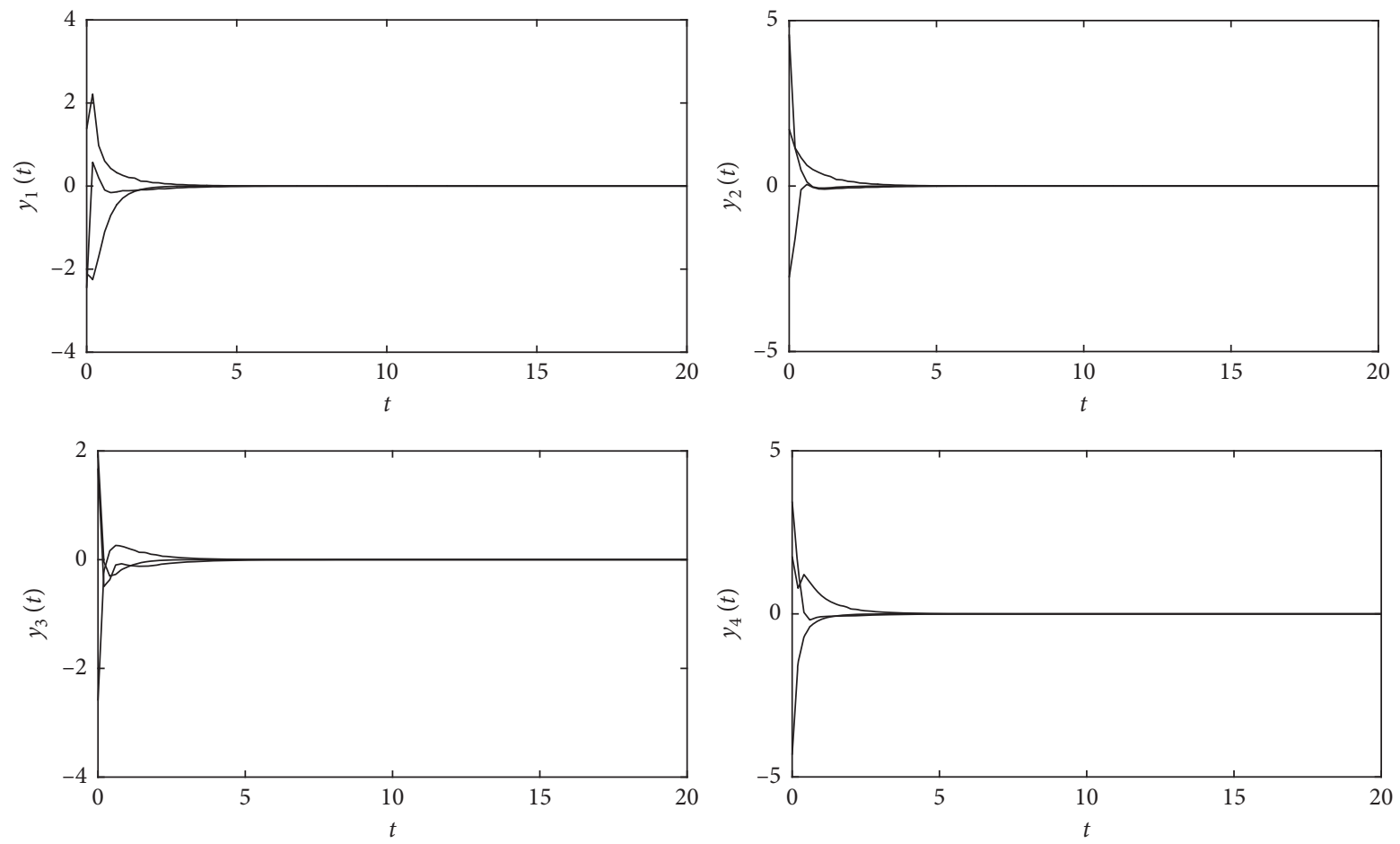

FIgURE 2: All the trajectories of the error systems $y_{i}(t)(i \in[1: 4])$ approach 0.

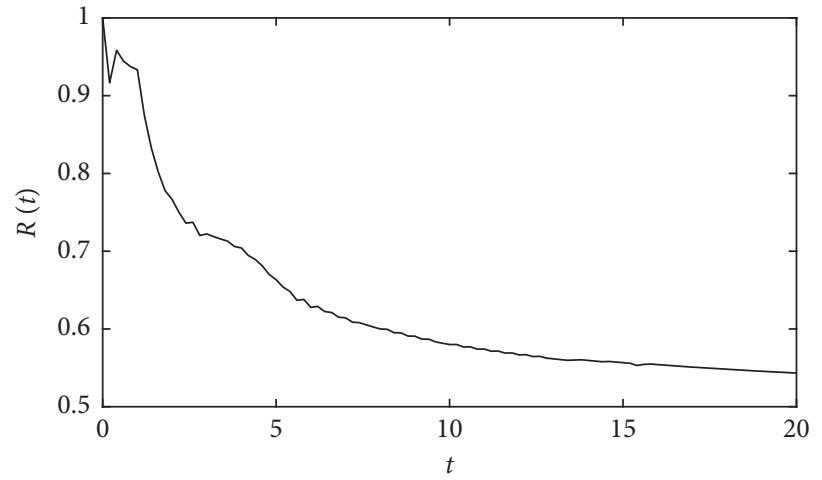

Figure 3: The trajectory of $R(t)$ for $t \leq 20$.

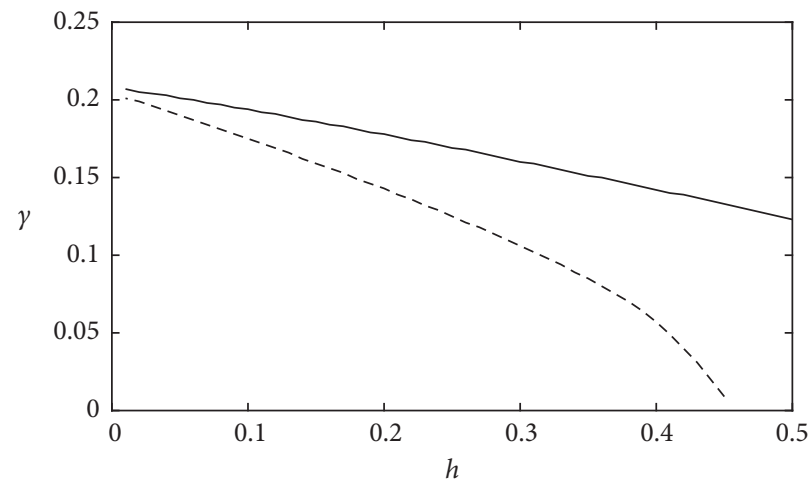

- Theorem 1

- - - Theorem 2

Figure 4: The maximum $\gamma$ which allows the existence of feasible solutions for Theorems 1 (the solid curve) and 2 (the dashed curve), when the sampling period $h$ is changing from 0.01 to 0.5 . 
conservatism, which is demonstrated by a numerical example.

\section{Data Availability}

The data used to support the findings of this study are available from the corresponding author upon request.

\section{Conflicts of Interest}

The authors declare that they have no conflicts of interest.

\section{References}

[1] J. Zhou and T. Chen, "Synchronization in general complex delayed dynamical networks," IEEE Transactions on Circuits and Systems I: Regular Papers, vol. 53, no. 3, pp. 733-744, 2006.

[2] W. Lu and T. Chen, "New approach to synchronization analysis of linearly coupled ordinary differential systems," Physica D: Nonlinear Phenomena, vol. 213, no. 2, pp. 214-230, 2006.

[3] J. Liang, Z. Wang, and X. Liu, "Exponential synchronization of stochastic delayed discrete-time complex networks," Nonlinear Dynamics, vol. 53, no. 1-2, pp. 153-165, 2008.

[4] Y. Li, J. Lou, Z. Wang, and F. E. Alsaadi, "Synchronization of dynamical networks with nonlinearly coupling function under hybrid pinning impulsive controllers," Journal of the Franklin Institute, vol. 355, no. 14, pp. 6520-6530, 2018.

[5] X. F. Wang and G. Chen, "Synchronization in small-world dynamical networks," International Journal of Bifurcation and Chaos, vol. 12, no. 1, pp. 187-192, 2002.

[6] C. W. Wu, "Synchronization in networks of nonlinear dynamical systems coupled via a directed graph," Nonlinearity, vol. 18, no. 3, pp. 1057-1064, 2005.

[7] J. Lu and J. Cao, "Adaptive synchronization in tree-like dynamical networks," Nonlinear Analysis: Real World Applications, vol. 8, no. 4, pp. 1252-1260, 2007.

[8] C. Huang, D. W. C. Ho, and J. Lu, "Synchronization analysis of a complex network family," Nonlinear Analysis: Real World Applications, vol. 11, no. 3, pp. 1933-1945, 2010.

[9] L. Li, D. W. C. Ho, and J. Lu, "A unified approach to practical consensus with quantized data and time delay," IEEE Transactions on Circuits and Systems I: Regular Papers, vol. 60, no. 10, pp. 2668-2678, 2013.

[10] H. Gao, J. Wu, and P. Shi, "Robust sampled-data $H_{\infty}$ control with stochastic sampling," Automatica, vol. 45, no. 7 , pp. 1729-1736, 2009.

[11] A. Albert, "Comparison of event-triggered and time-triggered concepts with regard to distributed control systems," Embedded World, vol. 2004, pp. 235-252, 2004.

[12] K. Åström and B. Bernhardsson, "Comparison of Riemann and Lebesque sampling for first order stochastic systems," in Proceedings of the 41st IEEE Conference on Decision and Control, Las Vegas, NV, USA, December 2002.

[13] P. Tabuada, "Event-triggered real-time scheduling of stabilizing control tasks," IEEE Transactions on Automatic Control, vol. 52, no. 9, pp. 1680-1685, 2007.

[14] J. Lunze and D. Lehmann, "A state-feedback approach to event-based control," Automatica, vol. 46, no. 1, pp. 211-215, 2010.

[15] X. Wang and M. D. Lemmon, "Event-triggering in distributed networked control systems," IEEE Transactions on Automatic Control, vol. 56, no. 3, pp. 586-601, 2010.
[16] X. Meng and T. Chen, "Event based agreement protocols for multi-agent networks," Automatica, vol. 49, no. 7, pp. 2125-2132, 2013.

[17] C. Huang, D. W. C. Ho, and G. Zhai, "Distributed stabilization of fuzzy networked systems with event-based sampling scheme," in Proceedings of the 2015 34th Chinese Control Conference (CCC), pp. 7475-7480, Hangzhou, China, July 2015.

[18] Z. Wu, Y. Wu, Z. Wu, and J. Lu, "Event-based synchronization of heterogeneous complex networks subject to transmission delays," IEEE Transactions on Systems, Man, and Cybernetics: Systems, vol. 48, no. 12, pp. 2126-2134, 2017.

[19] C. Huang, L. Li, and J. Lu, "Pinning Stabilization of Connected Neural Networks with Event-Based Couplings," in Proceedings of the 2014 International Conference On Mechatronics and Control (ICMC), pp. 2464-2469, Jinzhou, China, July 2014.

[20] L. Li, D. W. C. Ho, J. Cao, and J. Lu, "Pinning cluster synchronization in an array of coupled neural networks under eventbased mechanism," Neural Networks, vol. 76, pp. 1-12, 2016.

[21] Y. Li, "Impulsive synchronization of stochastic neural networks via controlling partial states," Neural Processing Letters, vol. 46, no. 1, pp. 59-69, 2017.

[22] X. Ge, Q. Han, X. Zhang, L. Ding, and F. Yang, "Distributed event-triggered estimation over sensor networks: a survey," IEEE Transactions on Cybernetics, vol. 50, no. 3, pp. 13061320, 2019.

[23] Y. Li, B. Li, Y. Liu, J. Lu, Z. Wang, and F. E. Alsaadi, "Set stability and stabilization of switched Boolean networks with state-based switching," IEEE Access, vol. 6, pp. 35624-35630, 2018.

[24] J. Yang, J. Lu, L. Li, Y. Liu, Z. Wang, and F. E. Alsaadi, “Eventtriggered control for the synchronization of Boolean control networks," Nonlinear Dynamics, vol. 96, no. 2, pp. 1335-1344, 2019.

[25] C. Huang, J. Lu, D. W. C. Ho, G. Zhai, and J. Cao, "Stabilization of probabilistic Boolean networks via pinning control strategy," Information Sciences, vol. 510, pp. 205-217, 2020.

[26] C. Ouyang and L. Li, "Event-based robust synchronization of Boolean control networks," IEEE Transactions on Circuits and Systems II: Express Briefs, vol. 67, no. 10, pp. 1969-1973, 2020.

[27] C. Huang, J. Lu, G. Zhai, J. Cao, G. Lu, and M. Perc, "Stability and stabilization in probability of probabilistic boolean networks," IEEE Transactions on Neural Networks and Learning Systems, vol. 32, no. 1, pp. 241-251, 2021.

[28] C. Zhou, L. Zemanová, G. Zamora-Lopez, C. Hilgetag, and J. Kurths, "Hierarchical organization unveiled by functional connectivity in complex brain networks," Physical Review Letters, vol. 97, no. 23, Article ID 238103, 2006.

[29] C. Huang, D. W. C. Ho, J. Lu, and J. Kurths, "Partial synchronization in stochastic dynamical networks with switching communication channels," Chaos, vol. 22, no. 2, Article ID 23108, 2012.

[30] B. Jiang, J. Lu, and Y. Liu, "Exponential stability of delayed systems with average-delay impulses," SIAM Journal on Control and Optimization, vol. 58, no. 6, pp. 3763-3784, 2020.

[31] S. Boyd, L. Ghaoui, E. Feron, and V. Balakrishnan, Linear Matrix Inequalities in System and Control Theory, Society for Industrial and Applied Mathematics, Cambridge, MA, USA, 1994.

[32] K. Gu, J. Chen, and V. Kharitonov, Stability of Time Delay Systems, Springer Science \& Business Media, Berlin, Germany, 2003.

[33] P. Naghshtabrizi, J. P. Hespanha, and A. R. Teel, "Exponential stability of impulsive systems with application to uncertain sampled-data systems," Systems \& Control Letters, vol. 57, no. 5, pp. 378-385, 2008.

[34] E. Fridman, "A refined input delay approach to sampled-data control,” Automatica, vol. 46, no. 2, pp. 421-427, 2010. 